\title{
Towards Improving Myocontrol of Prosthetic Hands: a Study on Automated Instability Detection
}

\author{
Roberto Meattini ${ }^{1}$, Markus Nowak ${ }^{2}$, Claudio Melchiorri ${ }^{1}$ and Claudio Castellini ${ }^{2}$
}

\begin{abstract}
Myocontrol is the control of an assistive device via the interpretation of the subject's intent using surface electromyography, and one paradigmatic instance of myocontrol is in upper-limb prosthetics applications. The reliability of this kind of control remains a key issue - effective and stable upper-limb myocontrol is one of the most interesting open problems in the field of human-robot interfaces and rehabilitation. In this work we focused on the myocontrol of a prosthetic hand while grasping: performing grasp actions only when, and exactly for the duration, the user desires, avoiding failures that can lead to frustrating or catastrophic results.

One specific step to improve stability in the myocontrol of prosthetic hands is the possibility to automatically detect the occurrence of a failure. For this purpose, the availability of an automatic "oracle" able to accomplish this work enables the possibility of self-adaptation of the myocontrol system - e.g. via on-demand model updates for incremental learning. According to this view, we performed an experiment using a simplified but still realistic grasping protocol involving four able-bodied expert myocontrol users, and we extracted features from a state-of-the-art commercial prosthetic hand to automatically identify instability in the myocontrol. The results show that a standard classifier is able to detect failures with a mean balanced error rate of $15.98 \%$ over the subjects that took part in the experiments. Our results can also be potentially applied in non-medical applications such as, e.g., teleoperation using extra-light interfaces.
\end{abstract}

\section{INTRODUCTION}

Myocontrol is the use of measurements of the muscle activity of a user, acquired by means of biosignals (traditionally surface electromyography, sEMG), for the control of assistive robots, or more in general for teleoperation in human-robot interaction scenarios [1]. Nowadays, despite the effort of the scientific community in the last decades, the control of rehabilitation and assistive devices is still afflicted by the problem of dealing with the issue of reliability. In general, this is due to the well-known difficulties in accurately detecting user intentions and relative timing, and to the inherent instability of the signals acquired from the human body; e.g. unexpected changes in the sEMG caused by sweating, electrodes displacement, muscle fatigue or specific postures and motions of the body segments [2]. When considering control of a prosthetic hand, the presence of unreliable myocontrol brings to unwanted behaviour like opening and closing of the fingers when not requested. In daily tasks

\footnotetext{
${ }^{1}$ Roberto Meattini and Claudio Melchiorri are with the Department of Electrical, Electronic and Information Engineering, University of Bologna, 40126 Bologna, Italy

${ }^{2}$ Markus Nowak and Claudio Castellini are with the Institute of Robotics and Mechatronics, German Aerospace Center (DLR), 82234 Oberpfaffenhofen, Germany.

Corresponding author: roberto.meattini2@unibo.it
}

based on hand manipulation, this causes unwanted grasp or release actions that can lead to catastrophic consequences in the worst cases (e.g. driving a car, carrying and manipulating fragile/hazardous materials, etc.) and to critical frustration of the user in the best cases.

The presence of instability in state-of-the-art myocontrol produces a poor reliability level that results in a rejection of this kind of systems by the users. However, at the same time, it has been extensively documented in literature how advanced myocontrol techniques are desired for the control of multifunctional prosthetic hands [3], [4], [5]. The reason why the scientific community has not yet been able to resolve this requirement-vs.-acceptance discrepancy can be found in the unstable nature of the above-mentioned signals [4], [6], but also - and not uncorrelated with - in a lack of designing appropriate testing protocols and framework to induce coadaptation between the user and the prosthetic device [7], [8].

We claim that a solution of the problem can be obtained introducing an incremental learning approach: based on the possibility of on-demand model updates, the control system can be taught new information leading to an interactive myocontrol paradigm [9], [10], [11]. This concept can be then integrated within a natural, simultaneous and proportional (s/p) control schema, where the possibility of updating should desirably be achieved in an autonomous and real-time fashion. Therefore, an automatic oracle, able to detect myocontrol instability, is the first step toward such an approach, that otherwise would require the presence of the subjective judgment of a human oracle (i.e., the experimenter or the subject), introducing a significant weakness in the whole paradigm. Following up our own previous work [12], here we propose a further advancement in this direction, especially by involving multiple subjects in a simplified experimental protocol, and improving the feature extraction and labelling techniques for the classification of myocontrol failures.

A myocontrol failure occurs every time the prosthetic hand performs a certain behaviour that does not coincide with the one expected by the user, i.e. it is in dissent with the user's intent. This comes from an instability of the myocontrol, which produces control outputs inconsistent with respect to the user will. The instability, in turn, occurs as a manifested consequence of a low robustness of the control system with respect to changes in the sEMG input signals (for a same given user's intent), that can be due to several aforementioned causes (sEMG inherent variability; influence of body postures and motions). In the light of these definitions, in our study we designed an extremely simplified, 
Visual Feedback

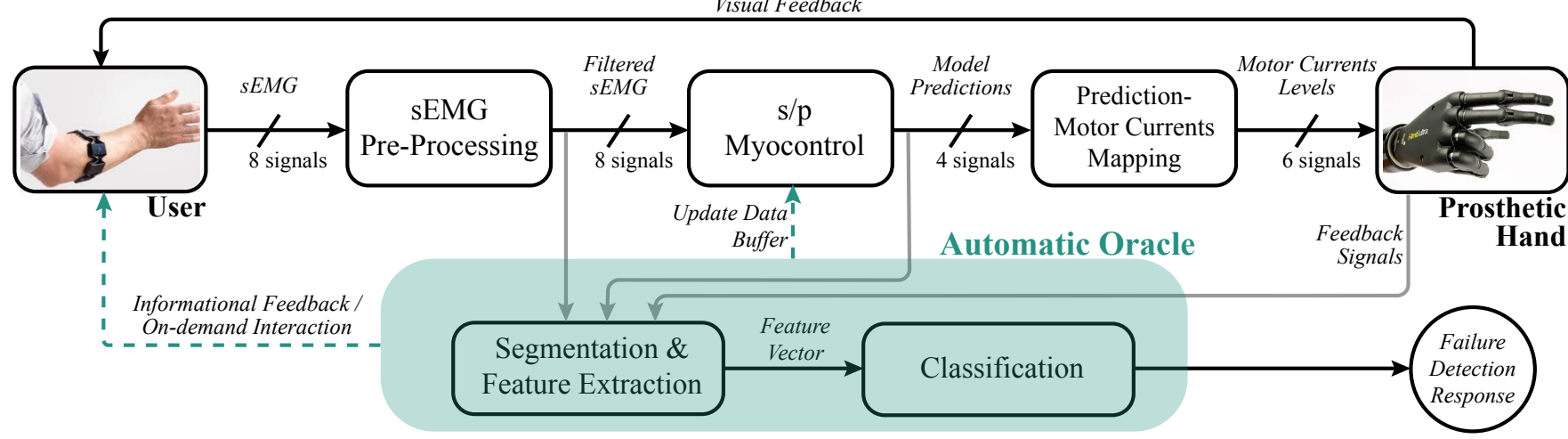

Fig. 1. Conceptual block diagram of the automatic oracle applied to the setup used in this work. The present study focuses on the possibility of detecting myocontrol failures using only a minimal set of feedback signals from the prosthetic device.

well-structured but still realistic grasp/carry/release task, in order to find specific features and evaluate the performance of an automatic detection of the myocontrol instability i.e. the objective of this work.

Grasping task experiments were performed engaging four able-bodied subjects equipped with a commercial sEMG bracelet and a multi-fingered six-Degrees-of-Freedom (DoFs) prosthesis. The participants were all experts in the usage of the state-of-the-art s/p myocontrol used in this work, since we wanted to detect failures rather than evaluating the quality of the myocontrol. Furthermore, by using video recordings of the experiments and the online information about the user's judgement on the occurrence of a failure provided by means of a Bluetooth button - we were able to label data instant by instant as myocontrol failure or success. The experimental results revealed that myocontrol failures could be detected with a mean balanced error rate over the subjects of $15.98 \%$ using a minimal set of feedback signals from the prosthetic hand. The paper is organized as follows: in Sec. II, the experimental setup and protocol are described, followed by the explanation of the procedures we used for labelling and classifying the myocontrol instability; Sec. III presents the results obtained over the subjects involved in the experimental sessions; finally Sec. IV outlines considerations and conclusions arising from our work.

\section{MATERIALS AND METHODS}

Fig. 1 depicts a block diagram that conceptually shows the idea of an automatic oracle for myocontrol failure detection, applied to the setup we used in this work. Referring to such a figure, it is possible to see how the automatic detection should be performed by a sort of "supervisor" (the oracle) that gathers data from the signal flow going from the user to the robotic hand, thereafter manipulates and interprets it (segmentation and feature extraction) and provides a response about the occurrence of a myocontrol failure (the classification output). This behaviour should be available online during the control of the prosthesis, also providing informational feedback to the user and/or demanding for an interaction if useful, and supplying specific buffered data to allow the $\mathrm{s} / \mathrm{p}$ myocontrol to update its model in the face

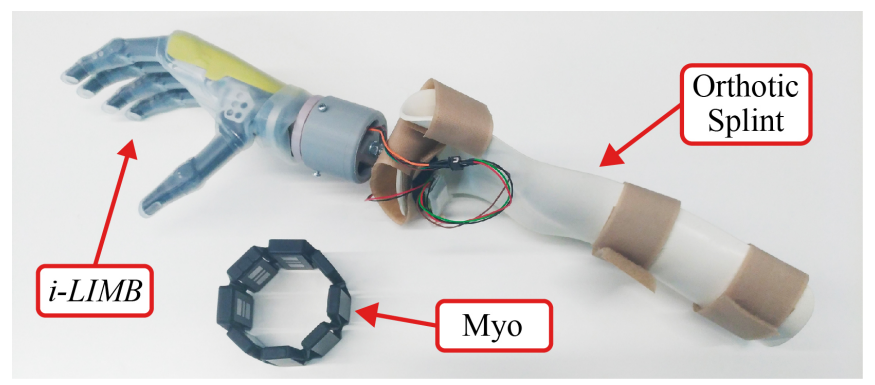

(a) Setup view.

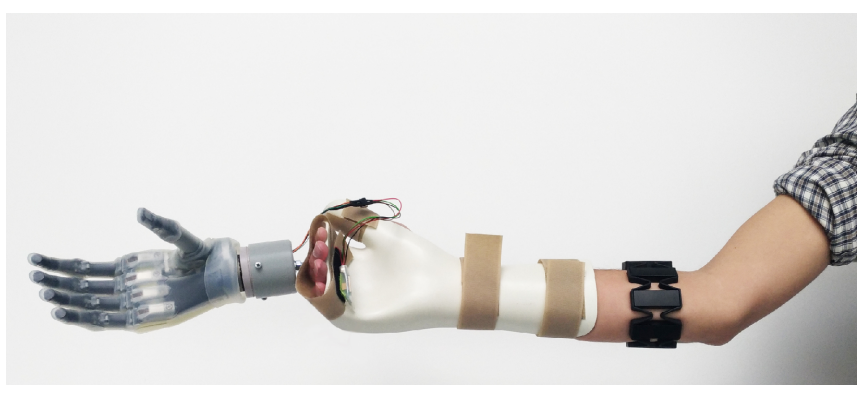

(b) The setup worn by one of the subjects.

Fig. 2. An overview of the experimental setup.

of new detected instabilities, according with an incremental learning paradigm. Relying on such architecture, in this work we focused on evaluating the possibility of classifying myocontrol failures using feature extracted from a minimal set of the prosthetic hand feedback signals.

\section{A. Setup Description}

In Fig. 2 we see the experimental setup used in this work. It consisted of a commercial orthotic splint specifically modified for the mounting of the prosthetic hands, an $i$-LIMB Revolution multi-fingered prosthetic hand manufactured by Touch Bionics [13] and a Myo bracelet by Thalmic Labs [14]. The Myo is equipped with eight integrated sEMG sensors, and it was worn by the subject in such a way to cover the full circumference of the users proximal forearm. The prosthetic 
hand has six step motors (two for the thumb and one for the rest of the fingers), each one of which can be directly controlled via current levels through a simple serial port protocol. The $i$-LIMB provides, for each motor, two kinds of feedback signals: the current reading and a "digit status" flag (opening, open, closing, closed and stalled).

The s/p myocontrol (see the related block in Fig. 1) was implemented using four parallel instances of Ridge Regression with Random Fourier Features (RR-RFF), a method already tested and used for myocontrol; the description of this particular Machine-Learning(ML)-based myocontrol algorithm lies outside of the article aims - for details refer to [9], [10], [15]. The input space of the myocontrol system was composed by the sEMG data, previously processed with a 1st-order Butterworth low-pass filter with cut-off at $1 \mathrm{~Hz}$. The output of the myocontroller was given by the outputs of the four RR-RFF instances, and it was directly fed as (proportionally scaled) current commands to the six motors of the prosthetic hand. Note that, in order to build the RR-RFF instances, the algorithm was trained by gathering sEMG data while the users performed specific actions in accordance with explicative visual stimuli administered by the $i-L I M B$ itself: i.e., the user followed the actions showed

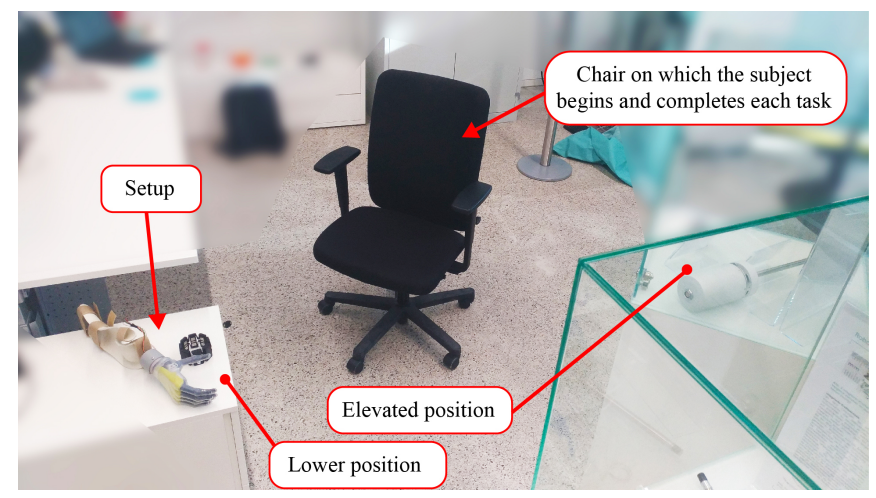

(a) Bird's-eye view on the laboratory organization for the experiment.
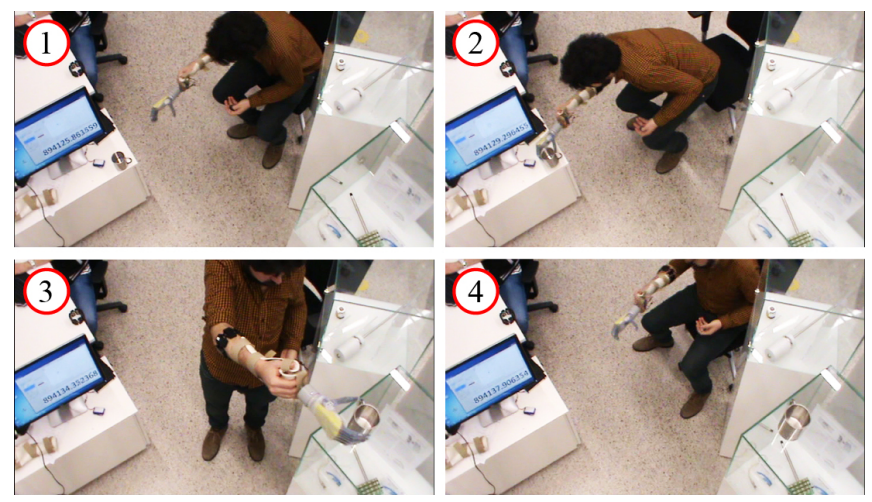

(b) Four frames from the video recording during the execution of a task: the subject (1) stood up and began the task, (2) grasped the cup in the lower position, (3) released the cup in the elevated position and (4) sat down completing the task.

Fig. 3. Experimental protocol overview. by the robotic hand while, automatically, the sEMG data was acquired in order to train the algorithm (for details see again [9]-[16], and refer to Subsec. II-B for information on the experimental procedure and specific actions). The reason why only four myocontrol prediction signals are used for the six DoFs of the prosthetic hand is that, for the grasp actions required in this study, the flexion of the middle, ring and little fingers are fully coupled (see Subsec. II-B). Furthermore, it is worth to note that the output of the RRRFF algorithm is a real number between 0 and 1 . In this relation, a threshold for the activation of the prosthetic hand was introduced (empirically set to 0.3 ) in order to let the users voluntarily trigger the prosthetic hand action.

\section{B. Experimental Protocol}

The experiment was performed in accordance with the Declaration of Helsinki and was approved by the Work Council of the German Aerospace Center. All participants were thoroughly informed about the experimental protocol and asked to sign an informed consent form. The subjects are four men between 28 and 45 years old, and all were experts in the usage of the s/p myocontrol used in this study (see Subsec. II-A). "Experts" means that they knew what is myocontrol and they already used the same setup at least two times in the last two months.

The experimental protocol was designed such that the sequence of actions required to the subjects during a grasping task were very simple and precisely defined. Furthermore, we also acquired video recordings of the whole experiment, and each user had a Bluetooth button available to signal when the myocontrol system would be failing: whenever the subject would realize that the prosthetic hand is not acting according to his intent, he should indicate this by pressing the wireless button.

All subjects performed two experimental sessions, each one composed by a training phase, in which the sEMG data was acquired to train the myocontrol ML algorithm, then followed by ten iterations of the grasping task. The training phase provided that the ML algorithm was trained with four different actions: "power grasp", "index pointing", "thumbsup" and "rest". During the training procedure, each action was trained while sitting with the elbow located on the chair arm rest. In particular the users were instructed to follow the indication given by the $i-L I M B$, which demonstrated to the user the sequence, timing and number of repetitions of the actions to be performed in this phase. Note that we explicitly decided to use only one repetition demonstrated by the user for the ML algorithm, in order to potentially stress instability and poor performance of the myocontrol system. The grasping task was composed by different steps. The subject started in a sitting position and, once indicated by the experimenter with a vocal signal, $(i)$ stood up, (ii) reached a close location where he had to grasp a mug using the "power grasp" action from a lower position (approximately $0.5 \mathrm{~m}$ from the floor), (iii) carried the mug to an elevated position $(\Delta d \approx 1 \mathrm{~m})$, (iv) released the mug and came back to the sitting position. The latter step determined the end 
of the grasping task. During the ten repetitions of the task within one experimental session, at every iteration the mug was moved in an opposite direction with respect to the previous one, i.e. moving the mug from the elevated to the low position or vice-versa. At this point the subject took a break of no less than 10 minutes, and then executed the second session in a similar way as the first one. Fig. 3 depicts an overview of the experimental protocol design and execution. It is worth to highlight that the specific design of the experimental protocol was chosen in order to insert instability stress factors within the execution of the tasks. Indeed, sources of myocontrol instability during Activities of Daily Living (ADL) can be principally due to: mental effort and lack of concentration of the user during the execution of simultaneous duties (i.e. controlling the prosthesis with muscle contraction while walking, sitting, etc.); usage of small data sets for the training of the ML-based myocontrol algorithm; user tiredness; and variations of the body posture (with a particular consideration for the posture of the arm from where the sEMG data is acquired) [17], [18], [19]. Therefore, the experimental task and protocol were designed in order to contain the following specific instability stress factors: increased possibility of misinterpretations of the ML myocontrol algorithm by training on multiple grasp types with only one repetition; training of the ML algorithm in a totally different body posture (in a sitting position with the elbow resting on the armrest) with respect to the one used during the task executions; variations of the body (and arm) posture in order to accomplish the task (standing, sitting, stretching in order to grasp and release in lower / higher locations, walk); and, finally, several task repetitions, which likely introduced non-negligible physical and mental efforts.

Note that it could also be possible that the occurrence of a failure prevented the completion of the task. In this case of catastrophic failure (i.e. the mug was dropped) the subject was instructed to return to the sitting position, the mug was returned to the original position and the attempted task was repeated. After the subject failed a second time in the very same situation, the ML model was updated. Since RR-RFF can be used incrementally, we added more information about the failed attempt to the database of the algorithm. For example, after the second failed attempt on release the mug in the elevated position, another repetition of the "rest" action would be trained to improve the control in this specific situation. Among all sessions, there were nine repeated attempts and updates were required only in two cases.

With this experimental protocol, by means of the video analysis and the opinion provided by the subjects, we were able to extract starting and ending times for the grasp actions, and to determine the instants in which a myocontrol failure occurred. In this context, we make the assumption that if we engage a human subject in a well-defined and extremely clear and simple task, the subject's intent will adhere to the actions required by the task. We can then claim that the subject's intent $i s$ the sequence of grasp actions requested during a task, and therefore with the aid of a video analysis we were

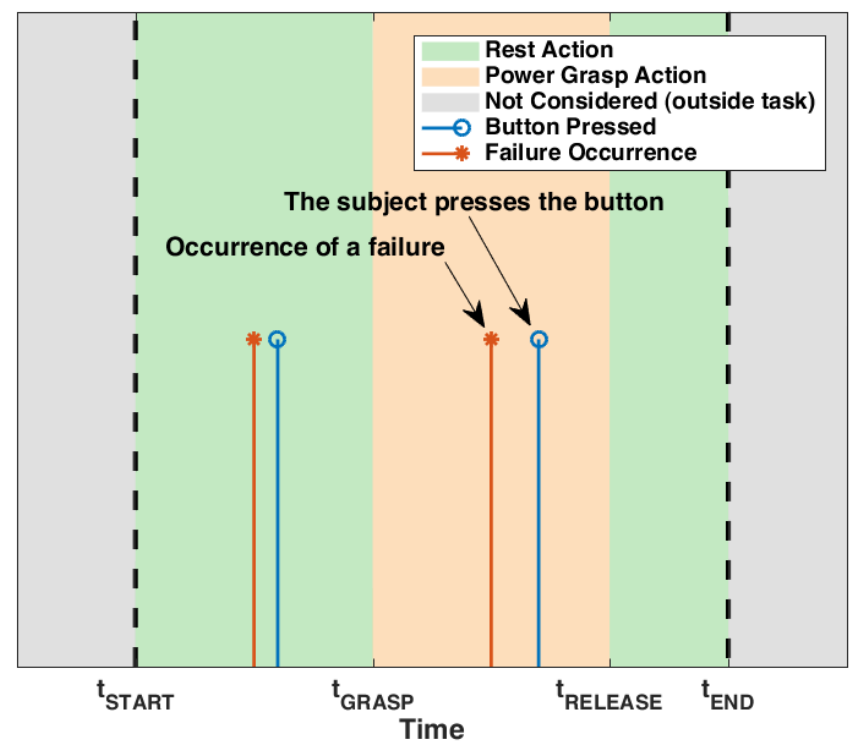

Fig. 4. A qualitative representation of the timing of a task, together with the user intent (red/green areas) and the failures. The latter were determined by video analysis (red), also exploiting the delayed information reported by the subject pressing the wireless button (blue).

able to instant by instant label the myocontrol performance as "failure" or "success".

\section{Labeling and Classification}

According to the design of the experimental protocol just described in Subsec. II-B, within every single task of the experimental session each subject was supposed to perform the following action sequence: to rest from the beginning of the task $\left(t_{S T A R T}\right)$ until grasping the mug; to grasp from this time $\left(t_{G R A S P}\right)$ until releasing it $\left(t_{R E L E A S E}\right)$; and to rest, again, from this time until the end of the task $\left(t_{E N D}\right)$. The starting and ending time of a task were input by the experimenter by pressing a key on the computer keyboard and vocally instructed to the subject, whereas $t_{G R A S P}$ and $t_{R E L E A S E}$ were determined manually by inspecting the video recording of each experiment (a timer was seen in the video, synchronized with the data recording). Therefore we had a reasonably reliable "user intent ground truth" that can be used to determine instability of the myocontrol and for labelling. A qualitative graph of the task and user intent timing is depicted in Fig. 4.

With the help of the video analysis, it was then possible to identify the instants of time in which a failure occurred. In particular, every performance of the prosthetic hand in accordance with the intent ground truth was considered as a "myocontrol success", whereas every time the prosthetic hand executed finger motions that disagree with the intent/ground truth, this was considered as a "myocontrol failure". To further help this analysis, the information of the user's opinion on the occurrence of a failure provided by pressing the wireless button was also used. However, in case of dissent of the information obtained through the video and by the user, the decision based on the mere video 
analysis was considered trustworthy, in order to remove online evaluation errors and delays introduced by the subjects (Fig. 4).

We used the outcome of the video analysis (i.e. instant of time of the failures) to label the feature extracted from the prosthetic hand feedback signals, in order to feed them into a classifier and evaluate the possibility of automatically determining an instability of the myocontrol (both false positives and negatives). The selection of the feature is based on the assumption that an instability of the myocontrol is reflected on the prediction outputs of the ML algorithm, displaying an oscillatory behaviour. Furthermore, according to the control mapping between the prediction signals and the motor currents for the control of the prosthetic hand (see Subsect. II-A), in order to actually make a failure happen the prediction signal has to necessarily cross the control threshold value of 0.3 for at least one of its four output values. This causes a change in the digit status of the prosthetic hand from open/opening to close/closing or vice-versa. Following these considerations, the "digit status" feedback available from the $i-L I M B$ was filtered to obtain a signal, named "filtered digit status", with only two possible values: "flexing" (replacing the closed/closing digit status) and "extending" (replacing the open/opening digit status). Then we used the counting of the variations of the filtered digit status value as the selected feature for the classification, computed by means of a running window. Therefore, we obtained a feature vector belonging to $\mathbb{N}^{4}(0$ stands for "extending", 1 stands for "flexing"). In particular, we tested three different window lengths, that is $0.5 s, 1 s$ and $3 s$, both without overlap and with an overlap of half the window length. The reason lies in the interest in evaluating the delays that the extraction of the feature would introduce in view of a real-time implementation of the instability detection.

The classification of the extracted feature was performed using a Support Vector Machine (SVM) classifier implemented by means of the LIBSVM library [20]. A nested cross-validation (CV) was applied to evaluate the performance of detecting myocontrol failures over the whole dataset (composed by 20 task executions, see Subsec. IIB) for each subject. In detail, the applied nested CV was composed by an inner 10-fold CV loop, where a grid-search was conducted for the selection of the best combination of the SVM classifier hyperparameters [21], and an outer 10fold CV loop in which the performance of the SVM model that won in the inner fold were tested on a separated external fold.

\section{EXPERIMENTAL RESULTS}

The evaluation of the classification performance of the extracted feature was performed by computing the confusion matrices and the balanced error rates (BER) over the different combinations of window length and overlap for the four subjects involved in the experiments.

Fig. 5 shows the matrices of the mean value over the four subjects of the true positive (TP), false positive (FP), false negative $(\mathrm{FN})$ and true negative $(\mathrm{TN})$ occurrence rates,

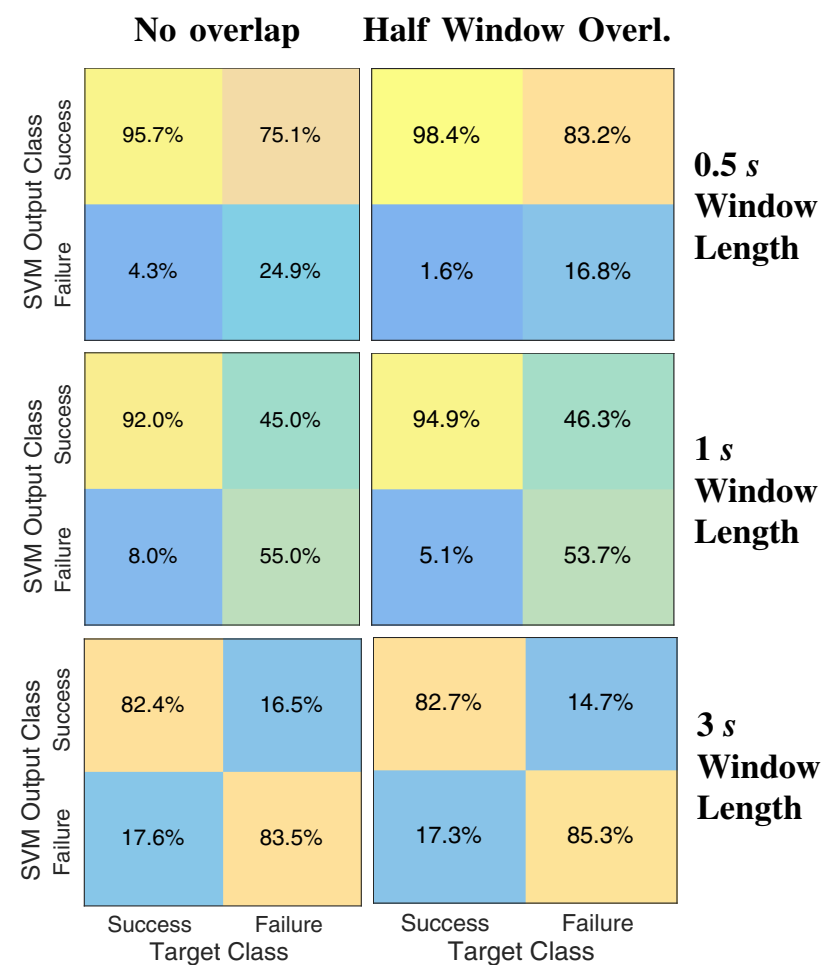

Fig. 5. The matrices of the mean occurrence rates (see Subsec. III) over the four subjects for the different combinations of window length and overlap.

the latter obtained from the computation of the confusion matrices. Specifically, the class "myocontrol success" (or simply "success") was considered as the positive condition (P), whereas the "myocontrol failure" (or, equivalently, "failure") was taken as the negative condition (N). According to this straightforward notation, the matrix of occurrence rates $M_{\%}$ was computed for each subject as

$$
M_{\%}=100\left[\begin{array}{cc}
\frac{N_{T P}}{N_{T P}+N_{F N}} & \frac{N_{F P}}{N_{F P}+N_{T N}} \\
\frac{N_{F N}}{N_{T P}+N_{F N}} & \frac{N_{T N}}{N_{F P}+N_{T N}}
\end{array}\right],
$$

where $N_{T P}, N_{F P}, N_{F N}, N_{T N}$ are the total number of TP, FP, FN and TN occurrences after the SVM classifier predictions, obtained during the outer loop 10-fold CV (see Subsec. II-C). The mean value over the four subjects of the elements of the matrices computed as in (1) is reported in Fig. 5 for the different combination of window length/overlap used for the extraction of the feature. Looking at the figure, it is possible to observe that, for window lengths of $0.5 \mathrm{~s}$ and $1 s$, the mean TP occurrence rate is greater then $90 \%$; however the mean $\mathrm{TN}$ occurrence rate remains under the $30 \%$. Furthermore, the mean FP occurrence rate is clearly greater than the $\mathrm{FN}$ rate, denoting that such selection of window lengths resulted in a consistent number of missed failure detections by the classier, especially marked for the length of $0.5 \mathrm{~s}$. On the other hand, the selection of a window length of $3 s$ reveals mean $\mathrm{TP}$ and $\mathrm{TN}$ occurrence rates greater than $80 \%$, therefore reporting for a low percentage of 
missed/wrong failure detections, with better results showed by the usage of the half window length overlap.

In order to consider the distribution of the failure detection performance among the subjects, we also evaluated the BER for the different window length/overlap combinations. The BER is again based on the confusion matrix elements, and is obtained as

$$
B E R=\frac{100}{2}\left(\frac{N_{F N}}{N_{T P}+N_{F N}}+\frac{N_{F P}}{N_{F P}+N_{T N}}\right),
$$

where the multiplication by 100 is present in order to obtain a percentage value. Fig. 6 shows the boxplot of the BER, grouped with respect to the feature extraction window lengths. Based on this graph, it is possible to see that better results are reported for the selection of a $3 s$ window (according to Fig. 5). Looking at the single boxplot related to the $3 s$ overlapped window in Fig. 6, it can also be observed that the distribution of the BER is skewed right (i.e., skewed "upward"), denoting a greater dispersion in case of values that fall within the upper quartile group. This is numerically confirmed by the statistical values reported in Tab. I, observing a mean BER value lower than the median value for the overlapped $3 s$ window. Furthermore, we can see that the highest data dispersion is reported by the choice of a $1 s$ window, indicating for a more pronounced variability between subjects in such case. This is visible looking at a BER IQR value of $14.87 \%$ and a standard deviation of $8.64 \%$ markedly higher compared to the others windowing methods (Tab. I); also, small whiskers (with respect to the IQR, Fig. 6) show a dispersion that is "spread" along the BER range of values. The lowest data dispersion is showed by the selection of the $0.5 \mathrm{~s}$ window length, reporting also the worst BER percentage result for the subjects engaged in the experiment. Additionally, we performed a two-way ANOVA with the factors Window Length and Window Overlap using the statistical tool provided by $R$ [22]. The test shows that the factor Window Overlap has no significant influence on the BER, $F(1,18)=0.014, p=0.908$. On the other hand, Window Length shows a significant influence on the BER, $F(2,18)=38.102, p<10^{-3}$. Following the ANOVA we performed a Tukey-Test to determine the pairwise interaction between the levels of Windows Length. The results show that all three pairwise interactions are significant, with $p<10^{-3}$, as indicated by the star symbols in Fig. 6.

\section{DISCUSSION AND CONCLUSIONS}

In this article we presented the results of a study for the improvement of the myocontrol of prosthetic hands with respect to the well-known reliability issue. We outlined the concept of an automatic oracle, i.e. a supervising agent that is able to classify when a myocontrol failure occurs, and to carry out an incremental learning paradigm to deal with myocontrol instability. Relying on this general framework, in this work we focused on the possibility of classifying the myocontrol instability using a minimal set of feedback signals from one of the most popular prosthetic hand

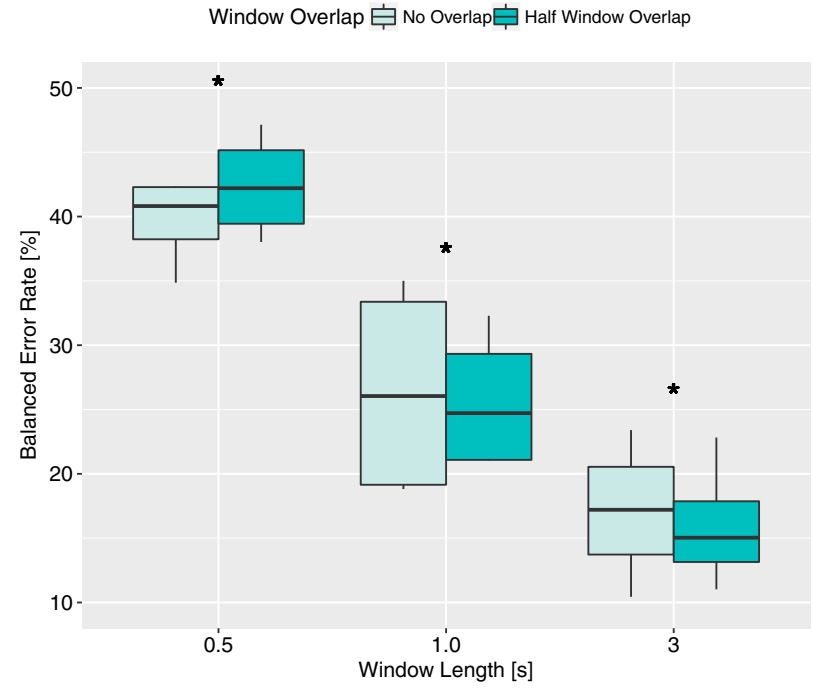

Fig. 6. Boxplot of the BER grouped by Window Length, for the two possible levels of Window Overlap. According to ANOVA, a star ("**" symbol) in proximity of a window length level group indicates statistically significant difference between said level and the remaining two levels of Window Length. There is no significant difference between the Window Overlap levels.

TABLE I

INDICATORS FOR THE BER DATA DISPERSION.

\begin{tabular}{c|lllll}
\hline \multirow{2}{*}{$\begin{array}{c}\text { Window Lengths } \\
{[\mathrm{s}]}\end{array}$} & $\begin{array}{l}\text { BER } \\
\text { Mean } \\
\text { Value } \\
{[\%]}\end{array}$ & $\begin{array}{l}\text { BER } \\
\text { Median } \\
\text { Value } \\
{[\%]}\end{array}$ & $\begin{array}{l}\text { BER } \\
\text { IQR } \\
{[\%]}\end{array}$ & $\begin{array}{l}\text { BER } \\
\text { Standard } \\
\text { Deviation } \\
{[\%]}\end{array}$ \\
\hline \multirow{2}{*}{0.5} & No Overlap & 39.71 & 40.82 & 5.2 & 3.52 \\
& $1 / 2$ Window Overlap & 42.39 & 42.21 & 6.85 & 4.17 \\
\hline \multirow{2}{*}{1} & No Overlap & 26.48 & 26.05 & 14.87 & 8.64 \\
& $1 / 2$ Window Overlap & 25.69 & 24.72 & 9.24 & 5.57 \\
\hline \multirow{2}{*}{3} & No Overlap & 17.06 & 17.21 & 8.86 & 5.64 \\
& $1 / 2$ Window Overlap & 15.98 & 15.04 & 7.07 & 5.03 \\
\hline
\end{tabular}

commercially available, the $i$-LIMB Revolution by Touch Bionics. To this purpose, we engaged four expert myocontrol users in a simplified and carefully designed experiment. The experiment consisted of twenty grasping tasks for each subject, in which we were able to identify the exact timing of myocontrol failure occurrences thanks to the information provided by the users and to an offline analysis of the experiment video recordings. This way, exploiting reasonable assumptions on the subjects' intent identification based on our experimental protocol design, it was possible to label a specific feature extracted from the prosthetic hand feedback signal as a success or a failure of the myocontrol system, in order to train and test a SVM classifier on the dataset gathered from the experiment. The selected feature was the counting of the changes from the extending status to the flexing status or vice-versa for each finger, obtained using three different running window lengths applied to the prosthetic hand feedback signal, with both possibilities of no overlap and half the window overlap. We reported for the best classification performances in the case of considering 
the feature set corresponding to a $3 s$ window, resulting in a mean BER of $15.98 \%$ over the four subjects. Furthermore this feature set - excluding the $0.5 \mathrm{~s}$ window case that reported the worst performance (median BER value greater than $40 \%$ ) - presents the lowest data dispersion (BER IQR $7.07 \%$ ) indicating a certain degree of repeatability in the classification accuracy at least for the preliminary results of this study.

The reported results positively highlight that information on the stability of a prosthetic myocontrol can be successfully obtained by gathering data from the assistive human-robot system itself. This incites future perspectives on myocontrol robustness and reliability improvements, e.g. inspiring further studies to implement online solutions for the failure detection. In addition, the classification of myocontrol failures by means of a basic feedback from the prosthetic hand - as done in this work - suggests that a few basic signals (i.e. sensors) and, in general, a low complexity suffice to enforce an effective automatic oracle.

The delay introduced by time segmentation and feature extraction, in view of an online implementation of the failure detection, cannot be overlooked — using the shortest timewindow of our experiment, $0.5 s$, showed non-satisfactory classification results. On the other hand, the delay introduced by the $3 s$ overlapping could be acceptable. Moreover, a fast response in the face of an instability occurrence is, in normal conditions, not necessary since the myocontrol failure would inevitably implicate an interruption of the normal usage of the assistive system by the user.

The results hereby presented represent a step towards a truly interactive prosthetic myocontrol, a system capable of autonomously identifying instabilities, intervening with proper updates and demanding for virtuous user-device interactions when necessary. This preliminary study is a starting point for a complete automatic oracle (Fig. 1) in a relevant context (e.g., with amputee patients). Indeed, about forthcoming future work, we will focus on improving the failure classification accuracy by first of all investigating on the extraction of more feature typologies, and performing tests on a group of participants that includes trans-radial amputees. For example, we consider interesting to evaluate the informational content of prosthetic hand-independent features extractable from the prediction outputs of the myocontrol ML algorithm; or, again, of user-only-dependent features extractable from the sEMG signals. We also want to work on a generalization of the failure definition and detection, for example considering the teleoperation of ideal and totally programmable artificial hands, as it is possible in the presence of virtual reality scenarios. In addition, such kind of studies are relevant also in relation to the possible application in non-prosthetic or -rehabilitation systems, i.e. in general teleoperation for human-robot interfaces.

\section{REFERENCES}

[1] R. Merletti, A. Botter, C. Cescon, M. Minetto, and T. Vieira, "Advances in surface EMG: Recent progress in clinical research applications," Critical reviews in biomedical engineering, vol. 38, no. 4, pp. 347-379, 2011.
[2] M. Santello, M. Bianchi, M. Gabiccini, E. Ricciardi, G. Salvietti, D. Prattichizzo, M. Ernst, A. Moscatelli, H. Jörntell, A. M. Kappers et al., "Hand synergies: integration of robotics and neuroscience for understanding the control of biological and artificial hands," Physics of life reviews, vol. 17, pp. 1-23, 2016.

[3] S. Micera, J. Carpaneto, and S. Raspopovic, "Control of hand prostheses using peripheral information," IEEE Reviews in Biomedical Engineering, vol. 3, pp. 48-68, October 2010.

[4] B. Peerdeman, D. Boere, H. Witteveen, R. Huis in 't Veld, H. Hermens, S. Stramigioli, H. Rietman, P. Veltink, and S. Misra, "Myoelectric forearm prostheses: State of the art from a user-centered perspective," Journal of Rehabilitation Research and Development, vol. 48, no. 6, pp. 719-738, 2011.

[5] M. Ison and P. Artemiadis, "The role of muscle synergies in myoelectric control: trends and challenges for simultaneous multifunction control," J. Neural Eng., vol. 11, 2014.

[6] A. Fougner, Ø. Stavdahl, P. J. Kyberd, Y. G. Losier, and P. A. Parker, "Control of upper limb prostheses: Terminology and proportional myoelectric control - a review," IEEE Trans Neur Syst Rehab Eng, vol. 20, no. 5, pp. 663-677, 2012.

[7] C. Castellini, R. M. Bongers, M. Nowak, and C. K. van der Sluis, "Upper-limb prosthetic myocontrol: two recommendations," Frontiers in Neuroscience, vol. 9, no. 496, 2015.

[8] M. Nowak, R. M. Bongers, C. K. van der Sluis, and C. Castellini, "Introducing a novel training and assessment protocol for pattern matching in myocontrol: case-study of a trans-radial amputee," in Proceedings of MEC - Myoelectric Control Symposium, 2017.

[9] A. Gijsberts, R. Bohra, D. Sierra González, A. Werner, M. Nowak, B. Caputo, M. Roa, and C. Castellini, "Stable myoelectric control of a hand prosthesis using non-linear incremental learning," Frontiers in Neurorobotics, vol. 8, no. 8, 2014.

[10] I. Strazzulla, M. Nowak, M. Controzzi, C. Cipriani, and C. Castellini, "Online bimanual manipulation using surface electromyography and incremental learning," IEEE Transactions on Neural Systems and Rehabilitation Engineering, vol. 25, no. 3, pp. 227-234, 2017.

[11] M. Nowak and C. Castellini, "The LET procedure for prosthetic myocontrol: Towards multi-DOF control using single-dof activations," PLoS ONE, vol. 11, no. 9, pp. 1-20, 92016.

[12] M. Nowak, S. Engel, and C. Castellini, "A preliminary study towards automatic detection of failures in myocontrol," in Proceedings of MEC - Myoelectric Control Symposium, 2017.

[13] " $i-L I M B \quad$ Revolution by Touch Bionics," www.touchbionics.com/products/active-prostheses/i-limb-revolution.

[14] "Thalmic Labs," www.thalmic.com.

[15] G. Patel, M. Nowak, and C. Castellini, "Exploiting knowledge composition to improve real-life hand prosthetic control," IEEE Transactions on Neural Systems and Rehabilitation Engineering, vol. 25, no. 7, pp. 967-975, 2017.

[16] D. Sierra González and C. Castellini, "A realistic implementation of ultrasound imaging as a human-machine interface for upper-limb amputees," Frontiers in Neurorobotics, vol. 7, no. 17, 2013.

[17] R. N. Khushaba, A. Al-Timemy, S. Kodagoda, and K. Nazarpour, "Combined influence of forearm orientation and muscular contraction on emg pattern recognition," Expert Systems with Applications, vol. 61, pp. 154-161, 2016.

[18] A. Fougner, E. Scheme, A. D. Chan, K. Englehart, and Ø. Stavdahl, "Resolving the limb position effect in myoelectric pattern recognition," IEEE Transactions on Neural Systems and Rehabilitation Engineering, vol. 19, no. 6, pp. 644-651, 2011.

[19] M. T. Wolf, C. Assad, M. T. Vernacchia, J. Fromm, and H. L. Jethani, "Gesture-based robot control with variable autonomy from the jpl biosleeve," in Robotics and Automation (ICRA), 2013 IEEE International Conference on. IEEE, 2013, pp. 1160-1165.

[20] C.-C. Chang and C.-J. Lin, "Libsvm: a library for support vector machines," ACM transactions on intelligent systems and technology (TIST), vol. 2, no. 3, p. 27, 2011.

[21] C.-W. Hsu, C.-C. Chang, C.-J. Lin et al., "A practical guide to support vector classification," 2003.

[22] R Core Team, R: A Language and Environment for Statistical Computing, R Foundation for Statistical Computing, Vienna, Austria, 2013. [Online]. Available: http://www.R-project.org/ 